Nicole Ritz $\cdot$ Roland A. Ammann

Carmen Casaulta Aebischer · Mathias Gugger

Katia Jaton · Ralph A. Schmid · Christoph Aebi

\title{
Failure of voriconazole to cure disseminated zygomycosis in an immunocompromised child
}

Received: 18 August 2004/ Revised: 22 November 2004/ Accepted: 24 November 2004/Published online: 5 January 2005

(C) Springer-Verlag 2005

\begin{abstract}
Voriconazole is increasingly used as a first-line agent for empirical antifungal therapy of prolonged febrile neutropenia in paediatric cancer patients. We describe the case of a 9-year-old patient with stage IV Burkitt lymphoma, who developed pulmonary and splenic zygomycosis while receiving voriconazole for persistent febrile neutropenia. The causative agent, Absidia corymbifera, was identified by broad-range fungal PCR in a lung biopsy sample. The patient was successfully treated with a combination of partial resection of the left upper lobe and antifungal therapy with high-dose liposomal amphotericin B followed by oral itraconazole as demonstrated by resolving pulmonary infiltrates on serial high resolution CT scans. Conclusion: This case emphasises that the lack of in vitro activity of voriconazole against zygomycetes is clinically relevant. Failure of voriconazole in suspected fungal infection should be investigated for the possibility of zygomycosis. Broad-range polymerase chain reaction may be able to identify the causative organism when cultures remain sterile.
\end{abstract}

N. Ritz · R. A. Ammann - C. C. Aebischer - C. Aebi $(\square)$ Department of Paediatrics, University of Bern, Inselspital, 3010 Bern, Switzerland

E-mail: christoph.aebi@insel.ch

Tel.: +41-31-6329487

Fax: + 41-31-6329468

M. Gugger

Institute of Pathology, University of Bern, Inselspital, Bern, Switzerland

R. A. Schmid

Division of General Thoracic Surgery, University of Bern, Inselspital, Bern, Switzerland

C. Aebi

Institute for Infectious Diseases, University of Bern, Inselspital, Bern, Switzerland

K. Jaton

Institute of Microbiology,

Centre Hospitalier Universitaire Vaudois (CHUV),

Lausanne, Switzerland
Keywords Absidia corymbifera $\cdot$ Broad-range polymerase chain reaction $\cdot M u c o r \cdot V o r i c o n a z o l e \cdot$ Zygomycosis

Abbreviations BAL: bronchoalveolar lavage - HRCT: high-resolution computed tomography - PET: positron emission tomography

\section{Introduction}

Febrile neutropenia is a common complication of chemotherapy in paediatric cancer patients. Standard management consists of broad-spectrum antibacterial therapy [7] and, if fever and neutropenia persist for more than 5 to 7 days, of antifungal therapy [7]. This strategy is based on the high risk of invasive fungal disease in prolonged febrile neutropenia, poor sensitivity of diagnostic procedures, and high case-fatality rate [6]. Amphotericin B has almost always been the drug of choice for empirical antifungal therapy; however, the disadvantages of conventional amphotericin B (infusion-related adverse events, nephrotoxicity, poor oral bioavailability) led to the development of new broad-spectrum antifungal agents, which include lipid formulations of amphotericin B, the echinocandins and new triazoles. Among the latter, voriconazole is of particular interest. Clinical trials in adults indicated comparable efficacy and a lower rate of adverse events in comparison with liposomal amphotericin B in neutropenia and persistent fever [17]. In addition, voriconazole can be administered orally. It is thus an attractive alternative to amphotericin B for empirical antifungal therapy in children, although no comparative trials have been conducted in this age group, and the clinical relevance of genetically determined variation of voriconazole metabolism has not been clarified [16]. Another potential disadvantage is the lack of antifungal activity against zygomycetes. Indeed, breakthrough 
zygomycoses have recently been reported in adults receiving voriconazole $[2,10,12]$. This is the first report of voriconazole failure in a child with disseminated zygomycosis.

\section{Case report}

A 9-year-old girl with stage IV Burkitt lymphoma diagnosed 5 months earlier developed fever after the last course of chemotherapy with methotrexate, cytarabine and dexamethasone $\left(10 \mathrm{mg} / \mathrm{m}^{2}\right.$ per day for 5 days). On physical examination, temperature was $40.2^{\circ} \mathrm{C}$, heart rate was $100 \mathrm{bpm}$, blood pressure was $100 / 60 \mathrm{mmHg}$, and $\mathrm{O}_{2}$ saturation was $96 \%$. There was a small skin papule at a former venipuncture site on the dorsum of the right hand. No other source of fever was found. Laboratory evaluation revealed a WBC of $0.1 \times 10^{9} / 1$, a platelet count of $11 \times 10^{9} / 1$, and a CRP level of $26 \mathrm{mg} / \mathrm{l}$. At that time, she had been severely neutropenic (WBC $\left.<0.5 \times 10^{9} / 1\right)$ for 17 days. Empirical therapy with cefepime and amikacin was started. Routine blood cultures grew Enterobacter cloacae and $\alpha$-haemolytic streptococci. On day 6 of persistent fever, the WBC was $0.3 \times 10^{9} / 1$, the platelet count was $30 \times 10^{9} / 1$, and the CRP level had risen to $158 \mathrm{mg} / 1$. A chest X-ray film showed a parenchymal consolidation in the left upper lobe suggestive of pneumonia and one of two blood cultures grew coagulase-negative staphylococci. Since these blood cultures were drawn from a central line and growth was reported after $60 \mathrm{~h}$ of incubation only, contamination was assumed. Nevertheless, antimicrobial therapy was changed to vancomycin, meropenem and clarithromycin. PCR for Mycoplasma pneumoniae from a nasopharyngeal aspirate and urinary Legionella pneumophila serotype 1 antigen test were negative. On day 10 of persistent fever, intravenous voriconazole $(6 \mathrm{mg} / \mathrm{kg}$ per dose every $12 \mathrm{~h}$ on day 1 and $4 \mathrm{mg} / \mathrm{kg}$ every $12 \mathrm{~h}$ thereafter) was added to the regimen. Highresolution computed tomography (HRCT) confirmed the presence of a consolidation in the left upper lobe (Fig. 1A), but failed to show a cavitary lesion, a "halo" sign or an "air-crescent" sign. Sonography of the abdomen was normal. On day 13 , the WBC count rose to $4.4 \times 10^{9} / 1$ (neutrophils $2.2 \times 10^{9} / 1$ ) and the CRP was $175 \mathrm{mg} / \mathrm{l}$. Serum galactomannan assays (Platelia Aspergillus, Bio-Rad) were negative on days 12, 18 and 24 . On day 17 , vancomycin and clarithromycin were discontinued. On day 19, the patient became afebrile and a HRCT scan demonstrated a decreased size of the consolidation. On discharge (day 26), the WBC was $6.8 \times 10^{9} / 1$ (neutrophils $5.8 \times 10^{9} / 1$ ) and the CRP level was $20 \mathrm{mg} / \mathrm{l}$. Voriconazole was continued orally for 4 weeks (until day 52). On day 36, post-chemotherapy evaluation demonstrated a small, persistent pulmonary lesion in the left upper lobe by HRCT. Positron emission tomography (PET) revealed a focal splenic lesion, which was confirmed by a repeat PET scan on day 52. Open spleen biopsy on day 61 showed granulomatous inflammation

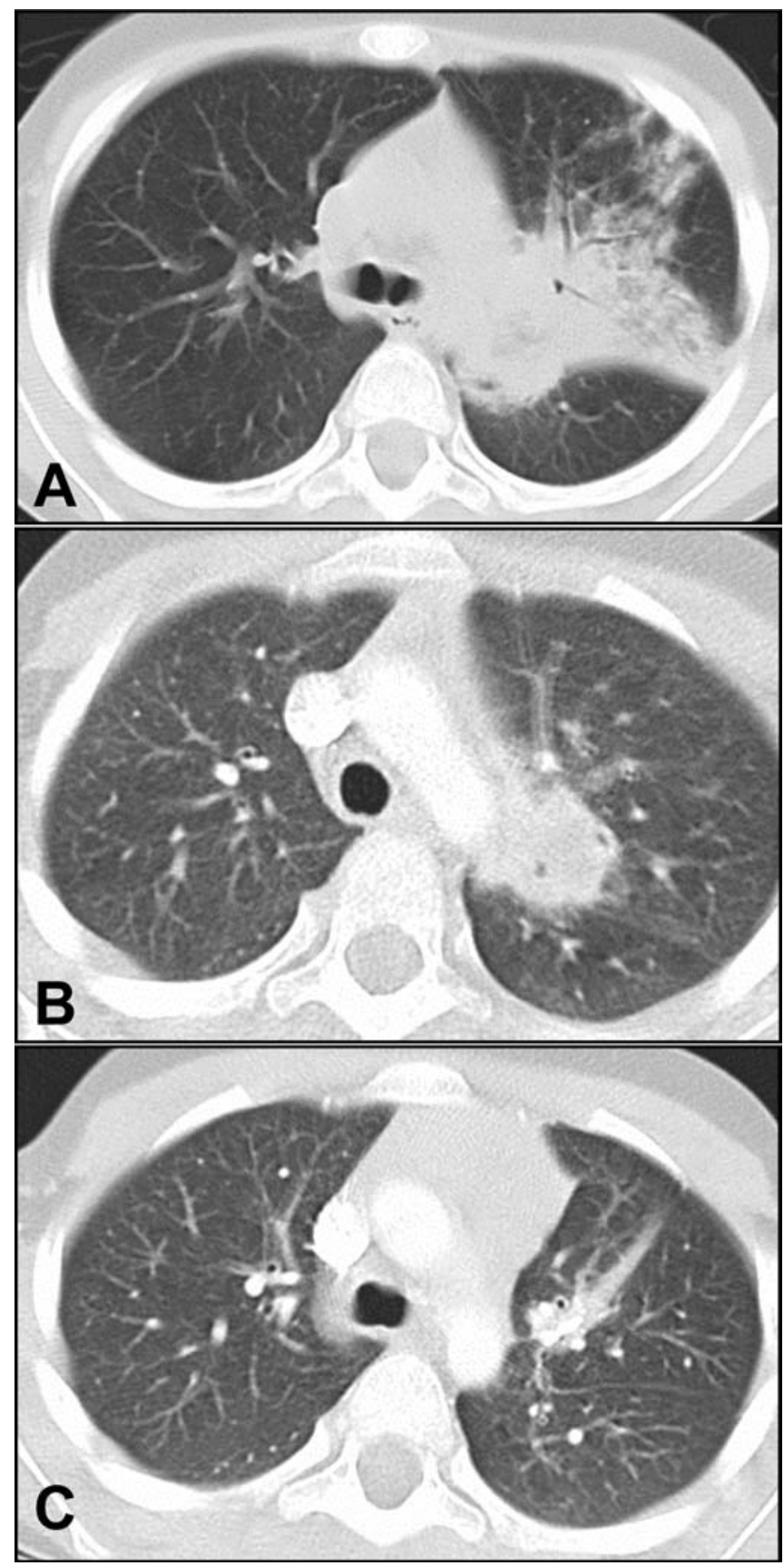

Fig. 1 HRCT of the lung. A Extensive left upper lobe infiltration on day 10. B A left hilar pulmonary abscess with inclusions of air (day 76). C Follow-up HRCT performed on day 110 shows residual changes following partial left upper lobe resection on day 81

with fungal elements. Hyphal morphology suggested zygomycosis. Fungal cultures remained negative. Liposomal amphotericin B (Ambisome) was started at $3 \mathrm{mg} /$ $\mathrm{kg}$ per day on day 66 and increased to $10 \mathrm{mg} / \mathrm{kg}$ per day on day 77. On day 76, an episode of haemoptysis occurred. HRCT showed a parenchymal abscess of $3 \mathrm{~cm}$ in diameter adjacent to the left hilus (Fig. 1B) and bronchoscopy revealed a $3 \mathrm{~mm}$, round, ulcerated plaque in the left upper lobe bronchus. Bronchoalveolar lavage 
(BAL) fungal cultures remained negative. Partial left upper lobe resection was performed on day 81. Zygomycosis was confirmed on a pulmonary biopsy specimen by histology (Fig. 2) and by broad-range fungal PCR targeting the 28S rRNA gene performed on DNA extracted from the embedded tissue. DNA sequencing of the amplified 28S rRNA fragment identified Absidia corymbifera. Fungal culture was negative. Follow-up HRCT and PET (both on day 92, 110 and 173) showed a gradually resolving pulmonary infiltrate (Fig. 1C) and persistent splenomegaly without focal lesions. On day 111 , antifungal therapy was changed to oral cyclodextrine-itraconazole ( $5 \mathrm{mg} / \mathrm{kg}$ once daily) for an additional 4 months.

\section{Discussion}

We describe the case of an immunocompromised child with disseminated $A$. corymbifera infection during empirical voriconazole treatment for prolonged febrile neutropenia. Recovery of two bacterial pathogens from blood cultures and early clinical improvement during treatment with antibacterial agents and voriconazole suggested that the initial pulmonary infiltrate was caused by a bacterial pathogen or a fungus susceptible to voriconazole (e.g. Aspergillus spp.), and that subsequent zygomycosis was a true breakthrough infection. Alternatively, occult zygomycosis could have been present from the beginning and temporary clinical improvement the result of concomitant bone marrow recovery. The term "breakthrough" infection is also applicable to this scenario [1]. Irrespective of the sequence of events, standard dose voriconazole failed to either treat or prevent A. corymbifera infection in this patient.

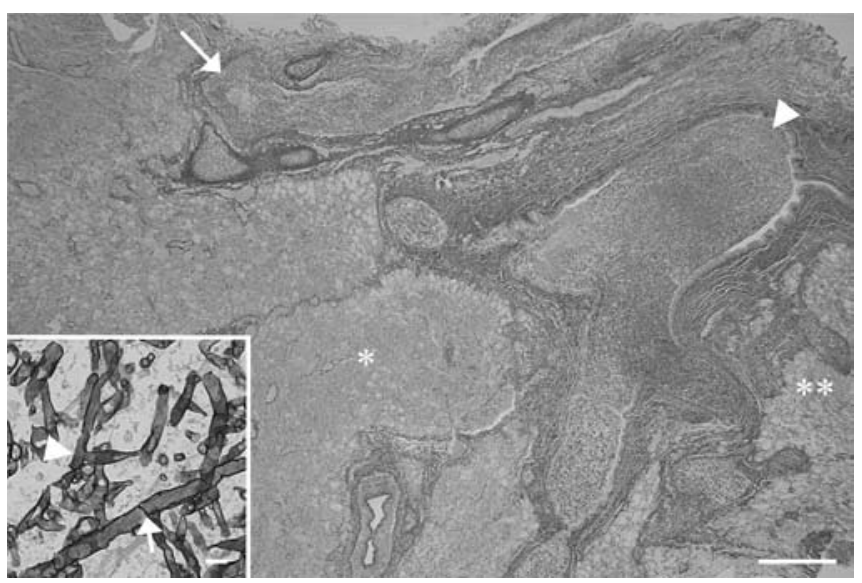

Fig. 2 The lumen of a bronchus (arrow) and the corresponding pulmonary artery (arrowhead) are occluded by loose aggregates of hyphae, visible in the insert. To the left of the pulmonary artery, the alveolar scaffold (asterisk) is less well delineated than to its right (double asterisk), corresponding to a necrotic area (silver methenamine stain, bar $=1000 \mu \mathrm{m})$. Insert: note the irregular diameter, the rare septa (arrow) and the nearly rectangular branching (arrowhead) of the thin-walled hyphae (silver methenamine stain, bar $=10 \mu \mathrm{m})$
Voriconazole demonstrates broad-spectrum antifungal activity in vitro. Zygomycetes, however, are mostly resistant [8]. Limited clinical evidence in adults indicates that voriconazole may also be ineffective against zygomycetes in vivo. As mentioned above, voriconazole and liposomal amphotericin B were compared in a recent multicentre trial of antifungal therapy in febrile neutropenia in adults [17]. Breakthrough invasive fungal infections occurred significantly less frequently in the voriconazole group, but there were two cases of zygomycosis in the voriconazole group and none in the liposomal amphotericin B group. Overall, voriconazole failed to demonstrate statistical equivalence to liposomal amphothericin B. Consequently, voriconazole was not approved by the United States Food and Drug Administration for empirical therapy of persistent fever during neutropenia. Failure of intravenous voriconazole in zygomycosis caused by Cunninghamella berthollettiae had previously been described in a 33-year-old male with acute myeloblastic leukaemia [12]. More recently, four cases of breakthrough zygomycosis in adult patients with allogeneic haematopoietic stem-cell transplants, who received voriconazole either as prophylaxis or empirical treatment, were reported from a single institution in the United States where zygomycoses were rarely seen before the advent of voriconazole [10]. A similar cluster of four cases of zygomycoses was reported from a transplant unit in France [2].

Here we report the first case of zygomycosis being diagnosed during voriconazole treatment in a child. The responsible organism, the ubiquitous mould $A$. corymbifera, has occasionally been implicated as a pathogen in immunocompromised children (Table 1). The reported locations of $A$. corymbifera infection in these cases were the nose, sinuses and skin. All patients were treated with conventional $(1 \mathrm{mg} / \mathrm{kg}$ per day) or liposomal $(1-15 \mathrm{mg} / \mathrm{kg}$ per day) amphotericin B. Two of five patients died, although death was attributable to A. corymbifera infection in one patient only. These cases are unlikely to reflect the entire spectrum of invasive A. corymbifera infection in children because fungal culture is insensitive for detection of zygomycetes in tissue specimens and BAL fluid. It is thus likely that many cases remain undiagnosed. The present case emphasises that nucleic acid detection methods such as pathogenspecific and broad-range fungal PCR followed by DNA sequencing of the amplified fragment may be useful tools for diagnosing zygomycoses. Documentation of A. corymbifera infection in our patient provided the rationale for continuing high-dose liposomal amphotericin $\mathrm{B}$ and offered the opportunity for switching to oral itraconazole, which exhibits in vitro activity against this particular organism $[3,8]$. Posaconazole would have been an alternative. This investigational azole has excellent in vitro activity against most zygomycetes including Rhizopus spp. [3], and both experimental [4] and anecdotal clinical evidence [14] suggest efficacy in vivo. No dosage recommendations are currently available for children. 
Voriconazole is an attractive agent for empirical antifungal therapy. This case, however, demonstrates that its lack of activity against zygomycetes is clinically relevant not only in adults, but also in children. Moreover, the case emphasises that aggressive diagnostic measures (e.g. BAL and tissue biopsy) for identification of a causative organism remain critical in prolonged febrile neutropenia with a pulmonary infiltrate, even if the obvious advantages of voriconazole encourage its empirical use. Finally, the case illustrates that cases of clinical failure of voriconazole should be investigated thoroughly for the possibility of zygomycosis, ideally by applying broad-range fungal PCR in culture-negative patients.

\section{References}

1. Bennett JE, Powers J, Walsh T, Viscoli C, de Pauw B, Dismukes W, Galgiani J, Glauser M, Herbrecht R, Kauffman C, Lee J, Pappas P, Rex J, Verweij P (2003) Forum report: issues in clinical trials of empirical antifungal therapy in treating febrile neutropenic patients. Clin Infect Dis 36: S117-S122

2. Clement L, Salmon A, Mansuy L, Paczesny S, Bordigoni P (2004) Mucormycosis after allogeneic haematopoietic stem cell transplantation. Bone Marrow Transplant 33: S205

3. Dannaoui E, Meletiadis, Mouton JW, Meis JFGM, Verweij PE (2003) In vitro susceptibilities of zygomycetes to conventional and new antifungals. J Antimicrob Chemother 511: 45-52

4. Dannaoui E, Meis JF, Leobenberg D, Verweij PE (2003) Activity of posaconazole in treatment of experimental disseminated zygomycosis. Antimicrob Agent Chemother 47: 36473650

5. Gebhard F, Chastagner P, Maillot D, Kures L, Georges JL, Schmitt C, Bordigoni P, Sommelet D (1995) Evolution favorable d'une mucormycose orbitonasosinusienne compliquant le traitement d'induction d'une leucémie aiguë lymphoblastique. Arch Pédiatr 2: 47-51

6. Grigull L, Beier R, Schrauder, Kirschner P, Loening L, Jack T, Welte K, Sykora KW, Schrappe M (2003) Invasive fungal infections are responsible for one-fifth of infectious death in children with ALL. Mycoses 46: 441-446

7. Hughes WT, Armstrong D, Bodey GP, Bow EJ, Brown AE, Calandra T, Feld R, Pizzo PA, Rolston KV, Shenep JL, Young LS (2002) Guidelines for the use of antimicrobial agents in neutropenic patients with cancer. Clin Infect Dis 34: 730-751

8. Johnson EM, Szekely A, Warnock DW (1998) In-vitro activity of voriconazole, itraconazole and amphotericin B against filamentous fungi. J Antimicrob Chemother 42: 741-745

9. Leong KW, Crowley B, White B, Crotty GM, O'Brian DS, Keane C, McCann SR (1997) Cutaneous mucormycosis due to Absidia corymbifera occurring after bone marrow transplantation. Bone Marrow Transplant 19: 513-515

10. Marty FM, Cosimi LA, Baden LR (2004) Breakthrough zygomycosis after voriconazole treatment in recipients of hematopoietic stem-cell transplants. N Engl J Med 350: 950951

11. Parkyn T, McNinch AW, Riordan T, Mott M (2000) Zygomycosis in relapsed acute leukaemia. J Infect 41: 265-282

12. Rickerts V, Loeffler J, Böhme A, Einsele H, Just-Nübling G (2001) Diagnosis of disseminated zygomycosis using a polymerase chain reaction assay. Eur J Clin Microbiol Infect Dis 20: $744-745$

13. Ryan M, Yeo S, Maguire A, Webb D, O'Marcaigh A, McDermott M, Butler K, O’Meara A (2001) Rhinocerebral zygomycosis in childhood acute lymphoblastic leukaemia. Eur J Pediatr 160: 235-238 
14. Tobon AM, Arango M, Fernandez D, Restrepo A (2003) Mucormycosis (zygomycosis) in a heart-kidney transplant recipient: recovery after posaconazole therapy. Clin Infect Dis 36: 1488-1491

15. Wali YA, Al Lamki Z, Al Kindi H, Taqi AM, Shah W, Soliman H, Zackaria M, Al Okbi H (2001) Successful outcome of invasive nasal sinus zygomycosis in a child with relapsed acute lymphoblastic leukaemia due to liposomal amphotericin B. Mycoses 44: 195-199

16. Walsh TJ, Lutsar I, Driscoll T, Dupont B, Roden M, Ghahramani P, Hodges M, Groll AH, Perfect JR (2002)
Voriconazole in treatment of aspergillosis, scedosporosis and other invasive fungal infections in children. Pediatr Infect Dis J 21: $240-248$

17. Walsh TJ, Pappas P, Winston DJ, Lazarus HM, Petersen F, Raffalli J, Yanovich S, Stiff P, Greenberg R, Donowitz G, Schuster M, Reboli A, Wingard J, Arndt C, Reinhardt J, Hadley S, Finberg R, Laverdiere M, Perfect J, Garber G, Fioritoni G, Anaissie E, Lee J (2002) Voriconazole compared with liposomal amphotericin B for empirical antifungal therapy in patients with neutropenia and persistent fever. N Engl J Med 346: 225-234 